\title{
Thyroid hormones modulate irisin concentrations in patients with recently onset hypothyroidism following total thyroidectomy
}

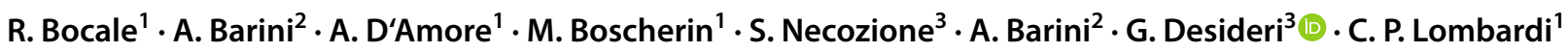

Received: 30 March 2020 / Accepted: 23 September 2020 / Published online: 14 October 2020

(c) The Author(s) 2020

\begin{abstract}
Purpose Irisin is a newly discovered adipo-myokine known for having significant effects on body metabolism. Currently, there is a discussion regarding the relation between thyroid function and irisin concentration. This study was designed to evaluate the influential role of levothyroxine replacement therapy on circulating levels of irisin in patients with recently onset hypothyroidism following total thyroidectomy.

Methods Circulating levels of thyroid hormones, irisin and other metabolic parameters, were assessed in 40 recently thyroidectomized patients ( 34 females, mean age $50.1 \pm 15.2$ years) at baseline (5-7 day after surgery) and after 2 months under replacement therapy with levothyroxine.

Results At baseline, circulating levels of thyroid hormones were indicative of hypothyroidism (TSH $12.7 \pm 5.0 \mu \mathrm{U} / \mathrm{mL}$, FT3 $1.9 \pm 0.7 \mathrm{pg} / \mathrm{mL}$, FT4 $8.7 \pm 3.6 \mathrm{pg} / \mathrm{mL}$ ). Mean serum irisin concentrations significantly increased after 2 months under replacement therapy with levothyroxine (from $2.2 \pm 0.6$ to $2.9 \pm 0.6 \mu \mathrm{g} / \mathrm{mL}, p<0.0001$ ). Variations of circulating levels of irisin under levothyroxine replacement therapy were directly correlated with those of FT3 (Rho $=0.454, p=0.0033)$ and FT4 $(\mathrm{Rho}=0.451, p=0.0035)$. Multivariate regression analysis revealed that changes in thyroid hormones concentrations explained up to $10 \%$ of the variations of serum irisin levels under levothyroxine replacement therapy $\left(\mathrm{FT} 3 R^{2}=0.098\right.$, FT4 $R^{2}=0.103$ ).
\end{abstract}

Conclusion Our study suggests that levothyroxine replacement therapy mildly influences irisin metabolism in patients with recently onset hypothyroidism following total thyroidectomy.

Keywords Irisin $\cdot$ Levothyroxine $\cdot$ Hypothyroidism

\section{Introduction}

Irisin, a newly discovered hormone-like adipo-myokine, is the extracellular cleaved product of fibronectin type III domain-containing 5 (FDNC5) and is regulated by peroxisome proliferator-activated receptor gamma (PPAR $\gamma$ ) coactivator-1 alpha $(\mathrm{PGC1} \alpha)$ [1]. Many researchers have reported its significant influence on metabolism and thermogenesis, mostly by promoting the so-called "browning" of subcutaneous adipose tissues via elevation of uncoupling protein 1 , contributing to improvements in glucose homeostasis and insulin resistance [1]. According to this, irisin has been evaluated in different metabolic disorders, such as obesity, insulin resistance, metabolic syndrome, type II diabetes mellitus as well as in subjects with increased cardiometabolic risk [2-4].

Thyroid hormones play important roles in regulating basal metabolic rate and thermogenesis, which may also be 
affected by irisin. Therefore, thyroid function could directly or indirectly be related to irisin regulation or, vice versa, irisin could affect the thyroid. In this regard, it has been proposed that increased TSH might lead to increased adipogenesis and irisin could be produced to keep the fat distribution in balance in the increased white adipose tissue [5]. Irisin has been studied in patients with thyroid dysfunction with somewhat conflicting results [5-8]. Therefore, it remains to clarify whether the effects of thyroid-axis hormones on metabolism could be possibly mediated by irisin, also because the evidences from interventional human studies that would support a causal effect of thyroid axis on irisin concentrations are currently limited.

This study aimed to evaluate the influential role of replacement therapy with levothyroxine (L-T4) on circulating levels of irisin in patients with recently onset hypothyroidism following total thyroidectomy.

\section{Materials and methods}

A total of 40 thyroidectomized patients ( 34 females and 6 males, mean age $50.1 \pm 15.2$ years) were randomly selected among those enrolled in a recently published study from our group aiming to compare the effects of replacement therapy with either liquid or tablet formulations of L-T4 on mood states, self-perceived psychological well-being and thyroid hormone profile in patients with recently onset hypothyroidism following total thyroidectomy [9]. Briefly, enrolled patients initiated L-T4 replacement therapy 5-7 days after the total thyroidectomy. The dose of L-T4 was individualized on the basis of the body weight of patients (about 1.6 $\mathrm{mcg} / \mathrm{kg}$ of body weight per day). All participants were in good clinical conditions without evidence of relevant comorbidities. Indication for thyroidectomy was represented by multinodular goitre with normal thyroid function while patients treated for thyroid malignancy were excluded [9].

\section{Laboratory evaluations}

All patients, at baseline and then at 2 months of followup, underwent blood samplings after an overnight fasting period for determination of the circulating levels of thyroid hormones, serum irisin, glucose, insulin and creatinine and lipid profile. TSH, FT3, FT4 and insulin were measured by COBAS 600 (Electrochemiluminescence Technology, Roche Diagnostics, Mannheim, Germany); reference range for TSH was $0.35-3.2 \mu \mathrm{U} / \mathrm{mL}$; reference range for FT3 was $2.4-4.2 \mathrm{pg} / \mathrm{mL}$; reference range for FT4 was $8.5-16.5 \mathrm{pg} /$ $\mathrm{mL}$. The homeostasis model assessment of insulin resistance (HOMA-IR) index was calculated according to the following formula: fasting serum insulin $(\mathrm{mU} / \mathrm{L}) \times$ fasting plasma glucose $(\mathrm{mmol} / \mathrm{L}) / 22.5$. Estimated glomerular filtration rate (eGFR) was assessed by Cockcroft-Gault formula. Irisin concentrations were measured with an enzyme-linked immunosorbent assay (ELISA) kit (measurement range: $0.001-5 \mu \mathrm{g} / \mathrm{mL}$; sensitivity: $0.001 \mu \mathrm{g} / \mathrm{mL}$; intrassay variability: $4.86 \%$ for $0.678 \mu \mathrm{g} / \mathrm{mL}$ and $7.63 \%$ for $1.37 \mu \mathrm{g} / \mathrm{mL}$ : interassay variability: $9.6 \%$ for $0.532 \mu \mathrm{g} / \mathrm{mL}$ and $8.02 \%$ for $1.14 \mu \mathrm{g} / \mathrm{mL}$ ) according to the manufacturer's directions (Biovendor, laboratory medicine, Czech Republic).

\section{Statistical analysis}

Pre-post differences between the assessed variables were analysed with Wilcoxon Rank-Sign Test after reject hypothesis of normality of their distributions with the Shapiro-Wilk test. Spearman's nonparametric correlation (Rho) was used to evaluate correlations between variables. Coefficient of determination $\left(R^{2}\right)$ was estimated to evaluate the proportion of the variation in the dependent variable explained by univariate regression models. Multivariate regression models were performed to verify the role of potentially confounding variables. The assumption of normality of the distribution of the residuals (the differences between the observations and the estimated values) was evaluated with the Shapiro-Wilk test. A Statistical analysis was performed using SAS 9.4 (SAS 2002-2012 by SAS Institute Inc., Cary, NC, USA).

\section{Results}

Patients were all in good clinical conditions without relevant diseases. General characteristics of the study population are presented in Table 1. Eight patients (20\%) were obese (body mass index $>30 \mathrm{~kg} / \mathrm{m}^{2}$ ). Circulating levels of thyroid hormones at baseline were indicative of either subclinical or overt hypothyroidism (TSH $12.7 \pm 5.0 \mu \mathrm{U} /$ $\mathrm{mL}$, FT3 $1.9 \pm 0.7 \mathrm{pg} / \mathrm{mL}$, FT4 $8.7 \pm 3.6 \mathrm{pg} / \mathrm{mL}$ ) (Table 1 , supplementary figures 1, 2 and 3 ). The mean serum irisin concentration at baseline was $2.2 \pm 0.6 \mu \mathrm{g} / \mathrm{mL}$ (Table 1 and supplementary figure 4).

After 2 months under L-T4 replacement therapy, a significant improvement of thyroid hormone profile was observed in the general study population (Table 1) although a few patients did not achieve a completely satisfactory thyroid hormones replacement or retained the condition of hypothyroidism (supplementary figures 1, 2 and 3). Serum irisin levels significantly increased under L-T4 replacement therapy (Fig. 1 and supplementary figure 4). Circulating levels of both HDL cholesterol and glucose slightly but significantly increased during replacement therapy while triglycerides concentrations significantly decreased (Table 1). A slight but significant reduction of eGFR was observed after 2 months under replacement therapy (Table 1). 
Table 1 General characteristics of study population ( $n=40$ subjects)

\begin{tabular}{llll}
\hline & Baseline & 2 months & $p$ value* \\
\hline Gender (females/males) & $34 / 6$ & - & - \\
Age (years) & $50.1 \pm 15.2$ & - & - \\
BMI $\left(\mathrm{kg} / \mathrm{m}^{2}\right)$ & $26.5 \pm 5.1$ & $26.6 \pm 5.1$ & 0.821 \\
SBP $(\mathrm{mmHg})$ & $125.7 \pm 10.5$ & $126.0 \pm 8.5$ & 0.979 \\
DBP $(\mathrm{mmHg})$ & $76.9 \pm 6.4$ & $77.4 \pm 5.7$ & 0.439 \\
TC $(\mathrm{mmol} / \mathrm{L})$ & $4.7 \pm 0.9$ & $4.8 \pm 0.8$ & 0.574 \\
LDL-C $(\mathrm{mmol} / \mathrm{L})$ & $2.8 \pm 0.8$ & $2.9 \pm 0.6$ & 0.611 \\
HDL-C $(\mathrm{mmol} / \mathrm{L})$ & $1.3 \pm 0.4$ & $1.4 \pm 0.4$ & 0.045 \\
TG $(\mathrm{mmol} / \mathrm{L})$ & $1.3 \pm 0.6$ & $1.1 \pm 0.4$ & 0.0009 \\
Glucose $(\mathrm{mmol} / \mathrm{L})$ & $4.6 \pm 0.9$ & $5.1 \pm 0.6$ & 0.0007 \\
Insulin $(\mathrm{mU} / \mathrm{L})$ & $10.4 \pm 5.1$ & $10.0 \pm 5.0$ & 0.543 \\
HOMA-IR & $2.2 \pm 1.1$ & $2.3 \pm 1.3$ & 0.346 \\
TSH $(\mu \mathrm{U} / \mathrm{mL})$ & $12.7 \pm 5.0$ & $3.7 \pm 4.3$ & $<0.0001$ \\
FT3 $(\mathrm{pg} / \mathrm{mL})$ & $1.9 \pm 0.7$ & $2.9 \pm 0.5$ & $<0.0001$ \\
FT4 $(\mathrm{pg} / \mathrm{mL})$ & $8.7 \pm 3.6$ & $15.5 \pm 2.7$ & $<0.0001$ \\
eGFR $(\mathrm{ml} / \mathrm{min})$ & $86.7 \pm 26.9$ & $82.7 \pm 25.4$ & 0.0017 \\
Irisin $(\mu \mathrm{g} / \mathrm{mL})$ & $2.2 \pm 0.6$ & $2.9 \pm 0.6$ & $<0.0001$ \\
\hline
\end{tabular}

$B M I$ Body Mass Index, SBP Systolic Blood Pressure, DBP Diastolic Blood Pressure, TC Total Cholesterol, $L D L-C$ Low Density Lipoprotein Cholesterol, $H D L-C$ High Density Lipoprotein Cholesterol, $T G$ Triglycerides, HOMA-IR Homeostasis Model Assessment-Insulin Resistance, TSH Thyroid Stimulating Hormone, FT3 Free T3, FT4 Free T4, eGFR estimated glomerular filtration rate. Plus-minus values are means \pm SD

*Wilcoxon Rank-Sign Test

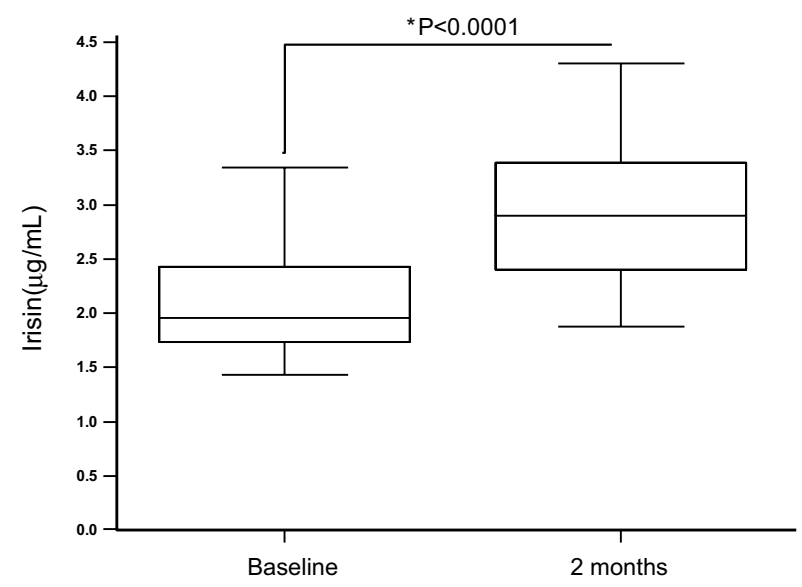

Fig. 1 Circulating irisin levels in patients with recently onset postthyroidectomy hypothyroidism at baseline and after 2 months under replacement therapy with levothyroxine $(* p<0.0001$, Wilcoxon Rank-Sign Test)

Changes in TSH levels after 2 months under L-T4 replacement therapy were significantly correlated with those of FT3 $(\mathrm{Rho}=-0.310, p=0.050)$ and FT4
(Rho $=-0.440, p=0.005)$, while these latter were correlated with each other $(\mathrm{Rho}=0.781, p<0.0001)$.

Variations of circulating levels of irisin under L-T4 replacement were correlated with those of FT3 $(\mathrm{Rho}=0.454$, $p=0.0033)$, FT4 $(\mathrm{Rho}=0.451, p=0.0035)$ and glucose levels (Rho $=0.463, p=0.0026$ ), with changes of HOMAIR $(\mathrm{Rho}=0.340, p=0.0317)$ and eGFR $(\mathrm{Rho}=-0.425$, $p=0.0063)$ and with age (Rho $=-0.362, p=0.0218)$. Univariate regression analysis revealed that changes of thyroid hormone levels explained up to $18 \%$ of the variations of serum irisin levels under L-T4 replacement therapy (FT3 $R^{2}=0.146, \beta=0.170 p=0.0152 ;$ FT4 $R^{2}=0.178 ; \beta=0.0367$ $p=0.007$ ) (Fig. 2a, b, respectively). Univariate regression analysis confirmed the relationship of changes of irisin levels with those of glucose $\left(R^{2}=0.110, \beta=0.146, p=0.037\right)$ and eGFR $\left(R^{2}=0.202, \beta=-0.027, p=0.0036\right)$ and with age
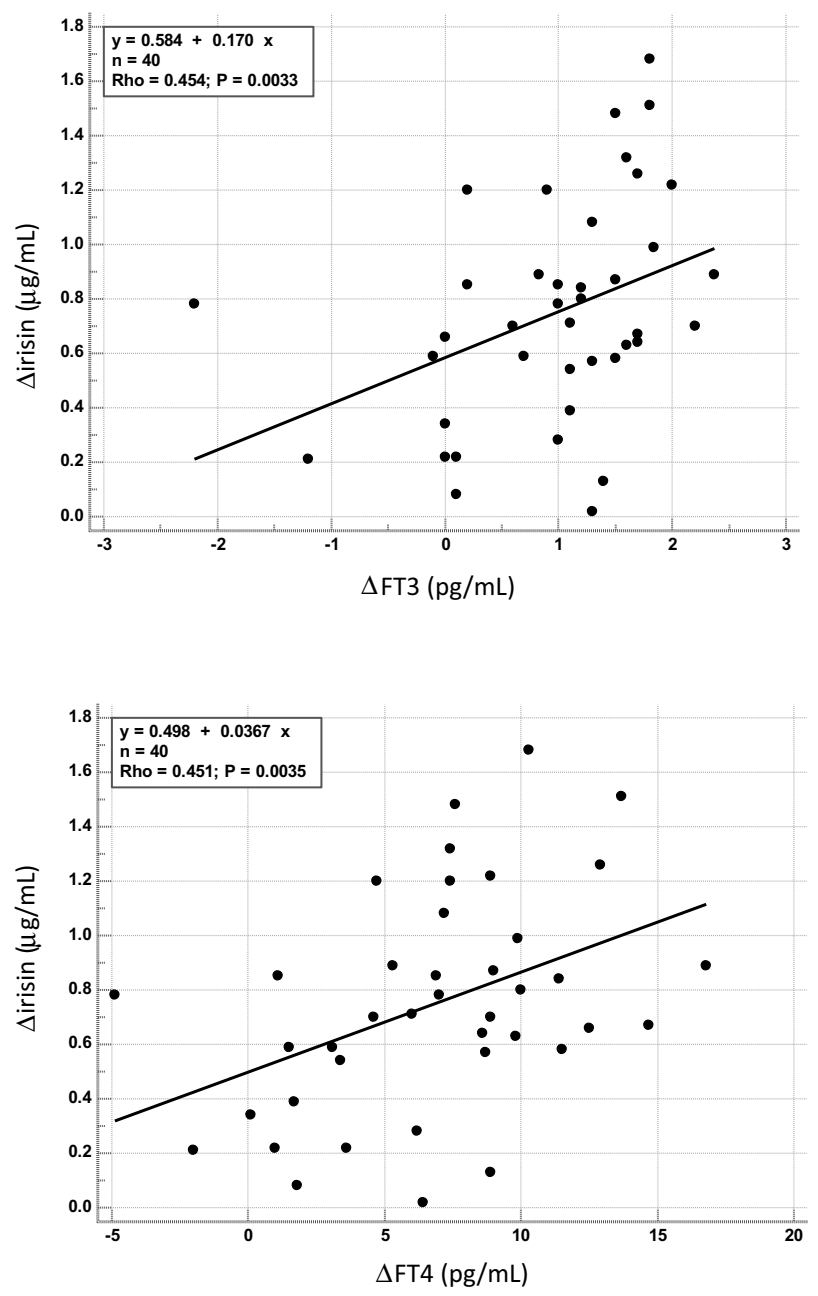

Fig. 2 Relationship between changes of irisin levels and changes of FT3 (upper panel) and FT4 (lower panel) in patients with recently onset post-thyroidectomy hypothyroidism after replacement therapy with levothyroxine 
$\left(R^{2}=0.133, \beta=-0.010, p=0.0208\right)$, but not with those of HOMA $\left(R^{2}=0.076, \beta=0.0868, p=0.085\right)$.

Multivariate regression analysis was performed to verify the role of potentially confounding variables which resulted associated with irisin variations in the Spearman's correlation analysis. Due to the collinearity between changes of FT3 and FT4, these variables were entered in two distinct models of multivariate analysis. Multivariate regression analysis confirmed the influential role of thyroid hormones on changes of irisin levels under L-T4 replacement therapy (Table 2). Change of eGFR was the only variable, other than thyroid hormones, which remained associated with changes of irisin levels under L-T4 replacement therapy (Table 2).

\section{Discussion}

The main interesting finding of our study was the significant increase of serum irisin levels under replacement therapy with L-T4 in patients with recently onset hypothyroidism following total thyroidectomy.

To date, the available data about the relationship between thyroid function and irisin are scarce and somewhat conflicting. In patients with overt hypothyroidism, circulating irisin levels have been described as either increased [5] or decreased [6, 8]. Similar discrepancies have been found also among studies which evaluate irisin levels in patient with subclinical hypothyroidism. Uc et al. [6] demonstrated that serum irisin levels are lower in patients with Hashimoto's thyroiditis in comparison to healthy subject and significantly increase following treatment to euthyroid state. More recently, Stratigou et al. [10] described significantly higher circulating irisin levels in patients with subclinical

Table 2 Multivariate regression analysis for predicting changes of irisin levels under levothyroxine replacement therapy

\begin{tabular}{llll}
\hline & Coefficient & $p$ & $R^{2}$ \\
\hline Model 1: FT3 & & \\
Changes of FT3 & 0.1468 & 0.0217 & 0.098 \\
Changes of eGFR & -0.02272 & 0.0181 & 0.105 \\
Changes of glucose & 0.08177 & 0.1840 & 0.031 \\
Age & -0.003670 & 0.3639 & 0.014 \\
Shapiro-Wilk test for normal distri- & $W=0.9770$ accept normality \\
bution & $(p=0.5781)$ & \\
Model 2: FT4 & \multicolumn{4}{c}{} \\
Changes of FT4 & 0.02909 & 0.0187 & 0.103 \\
Changes of eGFR & -0.02119 & 0.0252 & 0.092 \\
Changes of glucose & 0.09761 & 0.1047 & 0.047 \\
Age & -0.002843 & 0.4857 & 0.008 \\
Shapiro-Wilk test for normal distri- & $W=0.9691$ accept normality \\
bution & $(p=0.3361)$ \\
\hline
\end{tabular}

$e G F R$ estimated Glomerular Filtration Rate hypothyroidism which were not reverse by 6 months under L-T4 therapy. It was suggested that this might be attributed to the small number of treated patients, the short follow-up period and the variation of irisin not depending solely on TSH levels. On the other hand, two further studies described similar irisin levels in patients with subclinical hypothyroidism and in healthy control [7, 11]. In particular, Yasar et al. [11] enrolled patients with newly diagnosed subclinical hypothyroidism due to autoimmune thyroiditis (Hashimoto's thyroiditis). The authors hypothesized that thyroid tissuederived FNDC5/irisin [12] is released in the circulation as a result of the chronic inflammation of the thyroid [11]. On the other hand, Panagiotou et al. [7] evaluated the relationship between thyroid hormonal status and plasma irisin levels in cohort of thyroidectomized patients similar to those enrolled in our study. They found that irisin concentrations were not associated with thyroid-axis hormones cross-sectionally in either the overall cohort of thyroidectomized patients or in the euthyroid and/or subclinical hyperthyroid subgroups. The transient state of hypothyroidism due to levothyroxine withdrawal in patients with thyroid cancer was not associated with a significant change of circulating irisin concentrations. Finally, recombinant human TSH stimulation did not induce any significant changes in circulating irisin levels. Taken together, these findings seem to exclude any effect of TSH on irisin metabolism. On the other hand, the lack of data on the effect of replacement therapy on circulating levels of irisin does not allow to speculate on the possible influential role of thyroid hormones on irisin levels.

Our study sheds new light on this new research field providing a convincing evidence of an influential role of thyroid hormones on irisin metabolism. Indeed, we observed a significant increase of irisin levels under L-T4 treatment which were directly correlated with the increase of both FT3 and FT4 levels. The evidence of a direct relationship between changes of serum irisin levels and those of both FT3 and FT4 seems to suggest the ability of these hormones to modulate irisin metabolism even in the short-term. Since our patients were all euthyroid before thyroidectomy, the present results clearly suggest that the dynamic of the influential role of thyroid hormones on irisin metabolism is quite rapid. In this regard, previous evidence suggested that only long-lasting hypothyroidism is associated with a significant decreases of irisin, possibly as a result from muscle damage due to prolonged myopathy and leakage of irisin from damaged muscle cells, while short-term dysfunction was not associated with changes of irisin concentrations [8].

Our findings seem to be quite robust since they have been obtained in an homogeneous group of patients with recently onset post-thyroidectomy hypothyroidism, selected for having no concomitant clinical conditions potentially influencing irisin levels, including diabetes or chronic kidney disease [13]. Some discrepancies with the previous quoted studies 
could reflect differences in the enrolled populations, study design and methodological approach.

Although it may be premature, at this point, to conclude on the exact pathophysiological relationship between irisin and thyroid hormones, it is conceivable that irisin levels change, at least in part, according to the thyrometabolic status.

We also found that variations of circulating levels of irisin under L-T4 replacement were directly correlated with those of glucose. Although a positive relationship between serum irisin levels and metabolic risk factors has been already described in sedentary subjects [14], suggesting that metabolic factors, such as glucose or fatty acids, might represent important modulators of circulating irisin levels [15], the entity of this correlation is too small to speculate about an influential role of irisin on glucose metabolism, or vice versa, in our study population. According to this data interpretation, multivariate analysis did not reveal any significant influence of glucose levels on irisin metabolism.

Some limitations of the study should be mentioned. First, because of the study design [9], we cannot establish the circulating levels of irisin before thyroidectomy and thus, we cannot clarify the impact of surgical treatment itself on circulating levels of irisin. In addition, for obvious ethical reasons, our study did not consider a placebo arm in thyroidectomized patients, and thus we cannot completely exclude that a reduction in circulating levels of irisin would have occurred independently on replacement therapy. Second, although all patients underwent total thyroidectomy, we cannot exclude the persistence of some residual thyroid tissue which could have influenced the therapeutic dose required in post-surgical treatment $[16,17]$. Anyway, this potential bias likely did not significantly influence our findings since we did not consider the individual L-T4 doses but only the variation of thyroid hormones concentrations under L-T4 replacement therapy. Third, a few patients did not achieve a completely satisfactory thyroid hormones replacement or retained the condition of hypothyroidism, likely because of incompletely adequate adherence to the prescribed therapy. Anyway, this finding likely did not influence our data interpretation since we analyzed the relationship between individual changes of thyroid hormones and irisin concentrations independently on the achievement of an euthyroid status. Finally, given the current controversy around irisin assays and detection methods $[18,19]$, our finding allows us only to speculate about the pathophysiological relationship between thyroid hormones and irisin metabolism without at this moment clear and immediate clinical implications.

In conclusion, our results demonstrate that L-T4 replacement therapy mildly influences irisin metabolism in patients with recently onset hypothyroidism following total thyroidectomy.

Author contributions $\mathrm{RB}, \mathrm{AB}, \mathrm{GD}$ and $\mathrm{CPL}$ participated in study design and data analysis, and wrote the first draft of the manuscript. $\mathrm{AD}, \mathrm{MB}, \mathrm{SN}, \mathrm{AB}$ participated in data collection and analysis. All authors participated in manuscript preparation and approved its final version.

Funding Open access funding provided by Università degli Studi dell'Aquila within the CRUI-CARE Agreement. The authors received no financial support for the research, authorship, and/or publication of this article (none declared).

Data availability The datasets generated during and/or analysed during the current study are available from the corresponding author on reasonable request.

\section{Compliance with ethical standards}

Conflicts of interest The authors declared no potential conflicts of interest with respect to the research, authorship, and/or publication of this article (none declared).

Ethical standards The study was approved by the Catholic University of Sacred Heart Ethical Committee.

Research involving human participants and/or animals All procedures performed in this study were in accordance with the ethical standards of the institutional and/or national research committee and with the 1964 Helsinki declaration and its later amendments or comparable ethical standards.

Informed consent Informed consent was obtained from each participants included in the study.

Open Access This article is licensed under a Creative Commons Attribution 4.0 International License, which permits use, sharing, adaptation, distribution and reproduction in any medium or format, as long as you give appropriate credit to the original author(s) and the source, provide a link to the Creative Commons licence, and indicate if changes were made. The images or other third party material in this article are included in the article's Creative Commons licence, unless indicated otherwise in a credit line to the material. If material is not included in the article's Creative Commons licence and your intended use is not permitted by statutory regulation or exceeds the permitted use, you will need to obtain permission directly from the copyright holder. To view a copy of this licence, visit http://creativecommons.org/licenses/by/4.0/.

\section{References}

1. Moreno-Navarrete JM, Ortega F, Serrano M, Guerra E, Pardo G, Tinahones F, Ricart W (2013) Fernández-Real JM (2013) Irisin is expressed and produced by human muscle and adipose tissue in association with obesity and insulin resistance. J Clin Endocrinol Metab 98(4):E769-E778. https://doi.org/10.1210/jc.2012-2749

2. Panagiotou G, Mu L, Na B, Mukamal KJ, Mantzoros CS (2014) Circulating irisin, omentin-1, and lipoprotein subparticles in adults at higher cardiovascular risk. Metabolism 63(10):12651271. https://doi.org/10.1016/j.metabol.2014.06.001 
3. Gürel A, Doğantekin A, Özkan Y, Aydın S (2015) Serum apelin levels in patients with thyroid dysfunction. Int J Clin Exp Med 8(9): 16394-16398

4. Polak K, Czyzyk A, Simoncini T, Meczekalski B (2017) New markers of insulin resistance in polycystic ovary syndrome. J Endocrinol Investig 40(1):1-8. https://doi.org/10.1007/s4061 8-016-0523-8

5. Ateş İ, Altay M, Topçuoğlu C, Yılmaz FM (2016) Circulating levels of irisin is elevated in hypothyroidism, a case-control study. Arch Endocrinol Metab 60(2):95-100. https://doi. org/10.1590/2359-3997000000077

6. Uc ZA, Gorar S, Mizrak S, Gullu S (2019) Irisin levels increase after treatment in patients with newly diagnosed Hashimoto thyroiditis. J Endocrinol Investig 42:175-181

7. Panagiotou G, Pazaitou-Panayiotou K, Paschou SA, Komninou D, Kalogeris N, Vryonidou A, Mantzoros CS (2016) Changes in thyroid hormone levels within the normal and/or subclinical hyper- or hypothyroid range do not affect circulating irisin levels in humans. Thyroid 26(8):1039-1045. https://doi.org/10.1089/ thy.2016.0098

8. Zybek-Kocik A, Sawicka-Gutaj N, Wrotkowska E, Sowiński J, Ruchała M (2016) Time-dependent irisin concentration changes in patients affected by overt hypothyroidism. Endokrynol Polska 67(5):476-480. https://doi.org/10.5603/EP.a2016.0030

9. Lombardi CP, Bocale R, Barini A, Barini A, D'Amore A, Boscherini M, Bellantone R (2017) Comparative study between the effects of replacement therapy with liquid and tablet formulations of levothyroxine on mood states, self-perceived psychological wellbeing and thyroid hormone profile in recently thyroidectomized patients. Endocrine 55(1):51-59. https://doi.org/10.1007/s1202 0-016-1003-9

10. Stratigou T, Dalamaga M, Antonakos G, Marinou I, Vogiatzakis E, Christodoulatos GS, Karampela I, Papavassiliou AG (2018) Hyperirisinemia is independently associated with subclinical hypothyroidism: correlations with cardiometabolic biomarkers and risk factors. Endocrine 61(1):83-93

11. Yasar HY, Demirpence M, Colak A, Yurdakul L, Zeytinli M, Turkon H, Ekinci F, Günaslan A, Yasar E (2019) Serum irisin and apelin levels and markers of atherosclerosis in patients with subclinical hypothyroidism. Arch Endocrinol Metab 63(1):16-21. https://doi.org/10.20945/2359-3997000000106

12. Huh JY, Panagiotou G, Mougios V, Brinkoetter M, Vamvini MT, Schneider BE, Mantzoros CS (2012) FNDC5 and irisin in humans: I. Predictors of circulating concentrations in serum and plasma and II. mRNA expression and circulating concentrations in response to weight loss and exercise. Metab Clin Exp 61:17251738. https://doi.org/10.1016/j.metabol.2012.09.002
13. Maciorkowska M, Musiałowska D, Małyszko J (2019) Adropin and irisin in arterial hypertension, diabetes mellitus and chronic kidney disease. Adv Clin Exp Med 28(11):1571-1575. https://doi. org/10.17219/acem/104551

14. Moreno M, Moreno-Navarrete JM, Serrano M, Ortega F, Delgado E, Sanchez-Ragnarsson C, Valdés S, Botas P, Ricart W, Fernández-Real JM (2015) Circulating irisin levels are positively associated with metabolic risk factors in sedentary subjects. PLoS ONE 10(4):e0124100. https://doi.org/10.1371/journal.pone.01241 00

15. Kurdiova T, Balaz M, Vician M, Maderova D, Vlcek M, Valkovic L, Srbecky M, Imrich R, Kyselovicova O, Belan V, Jelok I, Wolfrum C, Klimes I, Krssak M, Zemkova E, Gasperikova D, Ukropec J, Ukropcova B (2014) Effects of obesity, diabetes and exercise on Fndc5 gene expression and irisin release in human skeletal muscle and adipose tissue: in vivo and in vitro studies. J Physiol 592(Pt 5):1091-1107. https://doi.org/10.1113/jphys iol.2013.264655 (PMID: 24297848)

16. Del Duca SC, Santaguida MG, Brusca N, Gatto I, Cellini M, Gargano L, Verga Falzacappa C, Frattaroli FM, Virili C, Centanni M (2015) Individually-tailored thyroxine requirement in the same patients before and after thyroidectomy: a longitudinal study. Eur J Endocrinol 173(3):351-357. https://doi.org/10.1530/EJE-15-0314

17. Jonklaas J, Bianco AC, Bauer AJ, Burman KD, Cappola AR, Celi FS, Cooper DS, Kim BW, Peeters RP, Rosenthal MS, Sawka AM, American Thyroid Association Task Force on Thyroid Hormone Replacement (2014) Guidelines for the treatment of hypothyroidism: prepared by the american thyroid association task force on thyroid hormone replacement. Thyroid 24(12):1670-1751. https ://doi.org/10.1089/thy.2014.0028

18. Albrecht E, Schering L, Buck F, Vlach K, Schober HC, Drevon CA, Maak S (2020) Irisin: still chasing shadows. Mol Metab 34:124-135. https://doi.org/10.1016/j.molmet.2020.01.016

19. Jedrychowski MP, Wrann CD, Paulo JA, Gerber KK, Szpyt J, Robinson MM, Nair KS, Gygi SP, Spiegelman BM (2015) Detection and quantitation of circulating human irisin by tandem mass spectrometry. Cell Metab 22(4):734-740. https://doi.org/10.1016/j. cmet.2015.08.001

Publisher's Note Springer Nature remains neutral with regard to jurisdictional claims in published maps and institutional affiliations. 